\title{
Airway Hyperresponsiveness in Asthma: A Better Understanding Yet to Yield Clinical Benefit
}

Ynuk Bossé ${ }^{1 *}$, Audrey Lee-Gosselin ${ }^{1}$, Louis-Philippe Boulet ${ }^{1}$ and Greg G King ${ }^{2}$

${ }^{1}$ Institut universitaire de cardiologie et de pneumologie de Québec (IUCPQ), Université Laval, Québec, Canada

${ }^{2}$ Woolcock Institute of Medical Research, University of Sydney, Australia

\begin{abstract}
Airway inflammation and hyperresponsiveness (AHR) are important features of asthma. Both inflammation and AHR are complex traits that can each originate from a plethora of factors, where every factor can be independent, interconnected and dispensable. This review examines the complexity of the indices that we use to assess airway responsiveness. These indices entail intricate information regarding the individual and the combined dynamic behavior of all the airways that constitute the tracheobronchial tree during the activation of airway smooth muscle (ASM). Because many factors other than ASM contractility can influence airway narrowing, the defects responsible for the manifestation of AHR are difficult to infer. New tests and technologies are being developed to decipher the meaning of the indices of airway responsiveness and have already leaped forward our understanding of AHR. This review also gives prominence to the concept of ASM plasticity.ASM mass and contractile capacity is not fixed over time. Several facets of inflammation can increase ASM force indirectly over a prolong period of time by causing tissue damage and repair, which ultimately leads of airway wall remodeling that embodies an enlargement of ASM mass. This could contribute to the fixed component of AHR. The gain of force due to inflammation can also be transient and conditional to the presence of inflammatory mediators that are capable of increasing the contractile capacity of ASM. This could contribute to the variable, and more readily modifiable, component of AHR. We are now aware that a multitude of muscle and non-muscle factors can contribute to AHR within an asthmatic individual, and that these factors are often times distinct between individuals. Consequently, the relative contribution of a single factor within a group of patients is usually very small. This is the reason why our ever-growing understanding of AHR in asthma does not quite yet avail patients.
\end{abstract}

Keywords: Inflammation; Airway smooth muscle; Asthma; Spirometry; Spasmogen

Abbreviations: AII: Angiotensin II; ACh: Acetylcholine; ATP: Adenosine-5'-Triphosphate; BDNF: Brain-Derived Neurotrophic Factor; CT-1: Cardiotrophin-1; GM-CSF: Granulocyte-Macrophage Colony-Stimulating Factor; 5-HT: 5-hydroxytryptamine (also called serotonin); HOCl: Hypochlorous Acid; IFN $\gamma$ : Interferon $\gamma$; IL: Interleukin; LIF: Leukemia Inhibitory Factor; LPA: Lysophosphatidic Acid; LT: Leukotriene; PAF: Platelet-Activating Factor; PDGF: PlateletDerived Growth Factor; PG: Prostaglandin; S1P: Sphingosine-1Phosphate; TGF $\beta 1$ : Transforming Growth Factor $\beta 1$; TNF $\alpha$ : Tumor Necrosis Factor $\alpha$; TXA : Thromboxane $\mathrm{A}_{2}$

\section{General Statements on Inflammation in Asthma}

Seminal and ongoing research has made it clear that the molecular signature of asthma is not unique [1]. The molecular abnormalities that have been described in asthma are rather heterogeneous, and those that lead to asthma symptoms also differ between affected individuals. This is not surprising, owing to the plethora of environmental factors that can trigger asthma. More than 500 individual triggers have been identified, including allergens [2], viruses [3], bacteria [3], pollutants [4], exercise [5], as well as occupational irritants [6,7] and sensitizers [8].

The pathognomonic symptoms of asthma are wheeze, breathlessness, chest tightness, and cough, which may be productive of phlegm. The symptoms are easily identified by clinicians, and establishing a diagnosis is relatively straightforward using reversibility airflow obstruction or measurement of airway responsiveness as suggested by current guidelines. However, the underlying mechanisms leading to inflammation and the subsequent development of symptoms are many. In fact, the physiopathology of asthma can vary from an individual to another. The inflammatory response is further convoluted by its variation in time. Asthma is often intermittent and often related to exposure to various environmental triggers. Over the years, the inflammatory response to environmental triggers can also change either in magnitude or in regard to the type of inflammation. Thus, the molecular basis of asthmatic inflammation is complex, heterogeneous and variable in time and severity.

\section{Complexity of Airway Responsiveness}

The degree of airway responsiveness is measured in research and clinical settings. Several tests are employed to assess airway responsiveness $[9,10]$. These tests have developed mainly for diagnostic purposes, as inducible airway obstruction is the key physiologic feature of asthma that can be measured in the laboratory [11]. Bronchoprovocation tests are meant to measure the effect of airway smooth muscle (ASM) activation on lung function. The tests are either direct or indirect, depending on whether they stimulate ASM directly via surface receptors or indirectly via mediators released from resident tissue. Direct tests usually consist of inhaling serial incremental doses

*Corresponding author: Ynuk Bossé, IUCPQ, Pavillon Marguerite-d'Youville, Y4186, 2725, chemin, Sainte-Foy, Québec, Qc, G1V 4G5, Canada, Tel: 418-6568711; E-mail: ynuk.bosse@criucpq.ulaval.ca

Received September 04, 2013; Accepted September 28, 2013; Published October 03, 2013

Citation: Bossé Y, Lee-Gosselin A, Boulet LP, King GG (2013) Airway Hyperresponsiveness in Asthma: A Better Understanding Yet to Yield Clinical Benefit. J Allergy Ther 4: 150. doi:10.4172/2155-6121.1000150

Copyright: @ 2013 Bossé $\mathrm{Y}$, et al. This is an open-access article distributed under the terms of the Creative Commons Attribution License, which permits unrestricted use, distribution, and reproduction in any medium, provided the original author and source are credited. 
of a spasmogen such as methacholine (MCh) [10]. Indirect tests, on the other hand, can take different forms, including: inhalation of mannitol, adenosine monophosphate and either hyper- or hypotonic saline; exercise or isocapnichyperpnoea; and bronchoprovocation with specific triggers such as an allergen. The indirect challenges aim at triggering inflammation, more specifically through the activation of mast cell degranulation, which then release inflammation-derived spasmogens to contract ASM. Both direct and indirect challenges provide valuable but different information. In fact, the degree of airway responsiveness in direct and indirect challenges does not always correlate [12]. In addition to being affected by the "twitchiness" of the airways, indirect challenges also depend on the subject's susceptibility to the chosen challenge; i.e. the propensity to release inflammationderived spasmogens in response to the challenge. The direct challenges are better measures of the 'twitchiness' of the airways, i.e. the propensity to respond upon activation of ASM. In terms of asthma diagnosis, the direct challenges are sensitive but not very specific. However, their high negative predictive value makes them useful to rule out asthma [10]. The indirect challenges are more specific. Although often less sensitive, they are better surrogates of the inflammatory state and therefore potentially more useful to evaluate and monitor the response to antiinflammatory drugs [13].

\section{Indices of Airway Responsivenessto Bronchoprovocative Challenges}

Spirometry is the most available and affordable tool used to measure airway responsiveness to either direct or indirect challenges in humans. Spirometry is also used to assess airway responsiveness to bronchodilators, but this will not be discussed in the present review. Spirometry appraises lung function by measuring volume and flow at the mouth during diverse breathing maneuvers. The most common breathing maneuver utilized to monitor the changes of lung function during or after a challenge is the forced expiratory volume in 1 second (FEV1). This is the volume of air that can be expelled during the first second of a maximal forced expiration from total lung capacity. A significant decline in FEV1 is indicative of a response to the agent used for the challenge.

The definition of airway hyperresponsiveness (AHR) is binary, i.e. the test is either positive or negative. Subjects either have AHR or they don't. The classification of a clinically significant response or 'positive response' to a challenge is arbitrary. The PC20, which is the provoking concentration of the inhaled spasmogen that causes a $20 \%$ decrease in FEV1, is often used as an index of airway responsiveness. The delivered dose, in $\mu \mathrm{mol}$ of $\mathrm{MCh}$ for example, can also be used to measure responsiveness. This is then called the PD20 (provoking dose). For MCh challenges for instance, if subjects have a PC20 to an inhaled concentration of $8 \mathrm{mg} / \mathrm{ml}$ or lower, they are considered to have AHR. This interpretation of the bronchoprovocation test is certainly sufficient for diagnostic purposes. This is because a positive response predicts clinically important factors such as the presence of symptoms, lung function variability and decline [14], treatment requirements and exacerbation risk [15].

However, airway responsiveness can also be treated has a continuous variable [16]. Some subjects may not have AHR, but still demonstrate a decline in FEV1 of less than 20\%. For research purposes, it is still useful to quantify their responsiveness to distinguish subjects that have a FEV1 decrease of $19 \%$ for instance, compared with $5 \%$. Clearly, the subject having a $19 \%$ decrease in FEV1 is more responsive than the subject whose FEV1 declines by only $5 \%$. The dose-response slope
(DRS=change in FEV1 as percent of baseline/dose or concentration of $\mathrm{MCh}$ ) is a continuous measure of responsiveness, irrespective of the presence or absence of AHR, which was defined by the arbitrary cutoff [17]. Higher DRS values represent greater responsiveness, which typifies asthmatic populations in comparison to non-asthmatic populations. Of note is that the DRS have a log-normal distribution within populations (Figure 1).

Bronchoprovocation tests can be conducted with concentrations or doses higher than that used for clinical purposes. The dose-response curves to such high-dose challenges to inhaled ASM agonists such as $\mathrm{MCh}$ are sigmoidal and can be analyzed for their position, slope and plateau [18] (Figure 2). Each of those indices of airway responsiveness carries different valuable information. The position is measured by the PC20 or PD20. The lower the PC20, the worse the responsiveness and the more leftward the position of the dose-response curve. The doseresponse slope (DRS) has been explained above. The maximal response is self-explanatory. It is assumed to represent the response obtained upon maximal activation of ASM. In fact, it is the response obtained with the highest dose. The maximal response cannot be quantified in hyperresponsive individuals because of the absence of a plateau. Highdose challenges are stopped at a 50\% decrease in FEV1 because of safety reasons. Potentially, hyperresponsive individuals will close off most of the lung leading to asphyxiation. This raises the possibility that the lack of the plateau is a physiologic demonstration of potentially clinically important risk of severe asthma attacks, if sufficient bronchial stimulus occurs.

\section{Non-muscle Factors in Airway Responsiveness}

Even if the responsiveness to an inhaled spasmogenre lies on ASM contraction, it is important to realize that many factors can affect the level of airway narrowing and the consequent gain of resistance to

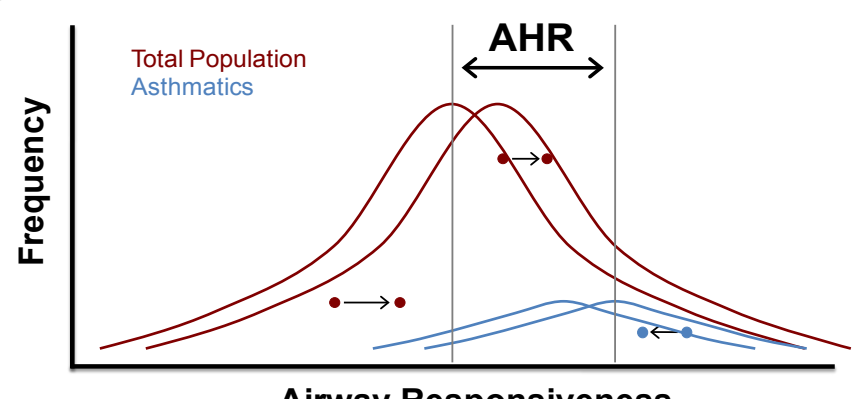

Airway Responsiveness

Figure 1: Some indices of airway responsiveness, such as the doseresponse slope, are continuous variables. Anyone can then be classified as hypo-, normo- or hyperresponsive, provided that arbitrary cutoffs are allocated. The graph demonstrates the theoretical frequency curves of the level of airway responsiveness in the total population (red) and the asthmatic population (blue). The difference between the average level of airway responsiveness between non-asthmatic and asthmatic populations is the airway hyperresponsiveness (AHR) that characterizes asthma. Notice that there is a lot of overlap; so that non-asthmatics can be hyperresponsive and asthmatics can be normoresponsive. The risk of suffering for asthma is lowered by a lot for someone hyporesponsive. AHR is thus perceived by many as a prerequisite to suffer asthma. The level of airway responsiveness is also not stable in time. The circles and arrows indicate how anyone in the population can be shifted along this frame. The dashed curves indicate how the average of the entire population can be shifted by different interventions. Whereas increasing inflammation can increase airway responsiveness (moves the population curve to the right), decreasing inflammation, by treating asthmatics with glucocorticoids for example, can decrease airway responsiveness (move the population curve to the left). The magnitude of those shifts is variable in between individual. 


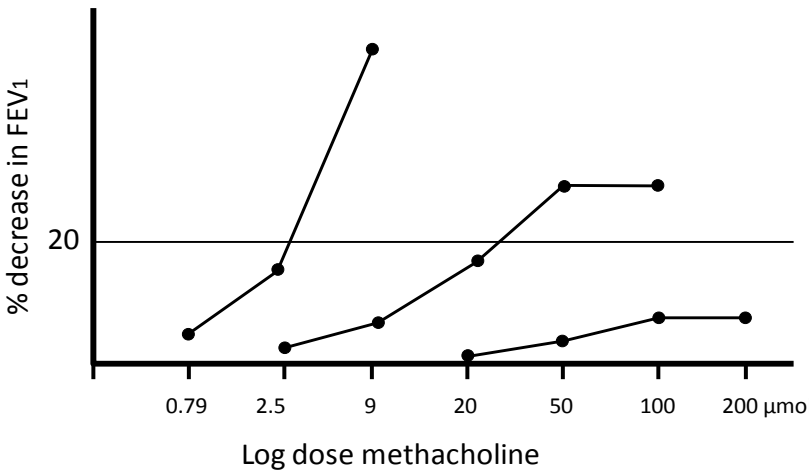

Figure 2: Typical dose-response curves in normals (right curve), mild asthmatics (middle curve) and moderate to severe asthmatics (left curve). Although responsiveness can be measured in normals, the curve does not reach the $20 \%$ decline in forced expiratory volume in $1 \mathrm{~s}$ (FEV1). Therefore, these individuals do not have a PD20; i.e. a provocative dose that decreases FEV1 by $20 \%$. However, several other indices can be used to assess their level of airway responsiveness. The position of the curves is a measure of sensitivity. A leftward displacement of the curve indicates hypersensitivity, i.e. that responsiveness can be measured at lower doses. The slope of the curves is a measure of airway reactivity. A greater dose-response slope (DRS) indicates a greater airway reactivity, i.e. that the magnitude of the response above the threshold point is greater. The plateau is a measure of the maximal achievable response. A higher plateau indicates a greater maximal achievable response. The dose-response curve in moderate to severe asthmatics is oftentimes characterized by the absence of a plateau.

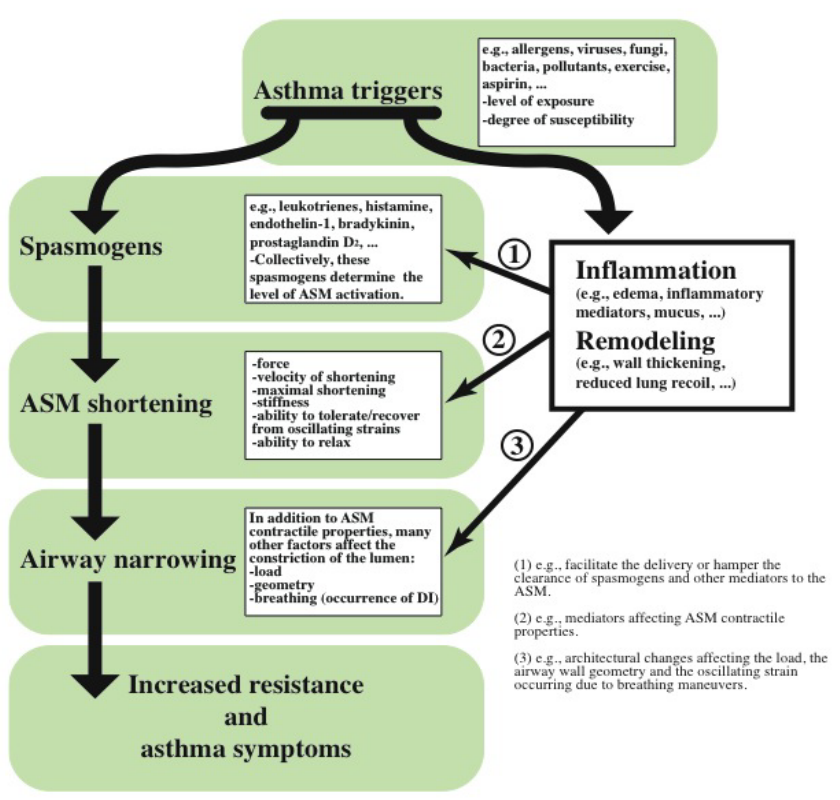

Figure 3: Diagram showing the airway responsiveness component on the left, the inflammatory/remodeling component on the right, and various ways by which they contribute and interconnect to affect asthma symptoms following exposure to asthma trigger(s). As seen in the diagram, a direct challenge with a spasmogen intervenes upstream, which means that airway responsiveness is affected by many downstream factors. The inflammatory/remodeling component can modify airway responsiveness (positively or negatively) eithe directly or indirectly by acting at different levels (enumerate 1 to 3 in the diagram) and by affecting both the size and the contractile capacity of ASM tissue. Reprinted and minimally adapted from Pulmonary, Pharmacology \& Therapeutics, 26/1, West AR, Syyong HT, Siddiqui S, Pascoe CD, Murphy TM, et al. (2013) Airway contractility and remodeling: Links to asthmasymptoms, pages 3-12, Copyright, with permission from Elsevier.

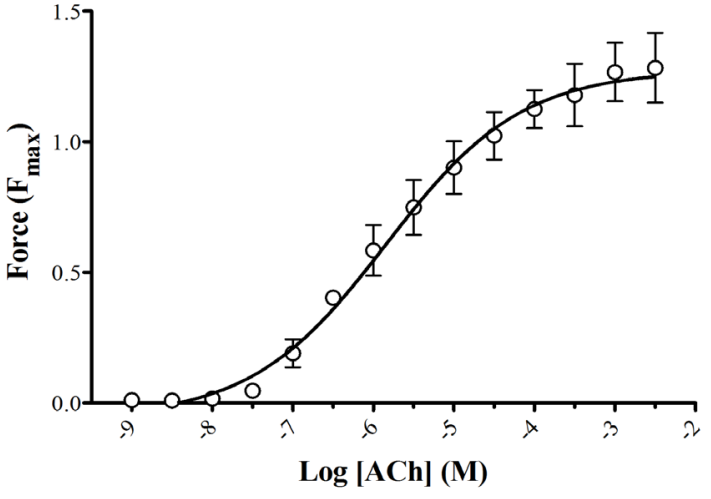

Figure 4: A concentration-response curve of isolated ovine tracheal strips stimulated to contract isometrically to acetylcholine. The curve demonstrates that greater is the level of ASM activation greater is the force. The force was normalized to $F_{\max }$, which is the force generated by an optimal electrical field stimulation. $n=4$.

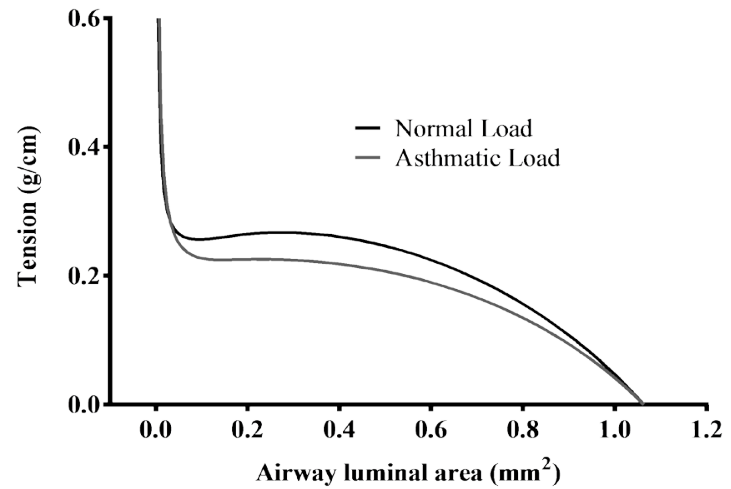

Figure 5: ASM load-airway luminal area curve, which describes the theoretical load that impedes ASM shortening during airway narrowing. The load is expressed in gram per $\mathrm{cm}$ of airway length. In this example, the normal (black) and asthmatic (grey) loads were calculated for an airway of the $10^{\text {th }}$ generation within a lung inflated to functional residual capacity (transpulmonary pressure of $5 \mathrm{~cm} \mathrm{H_{2 }}$ O). The loads take into account the transpulmonary pressure, the passive elastic elements of the airway wall, the distortion of the parenchyma surrounding the airway during ASM shortening, as well as the geometry of the airway generation analyzed [95]. The load is decreased in asthmatics because the thickening of the adventitia geometrically attenuates the coupling of the airway wall to the surrounding parenchyma [95]

airflow (Figure 3). First, the level of narrowing depends on ASM force. The force is predominantly determines by the level of ASM activation. A concentration-response curve of an isolated tracheal ASM strip is shown in Figure 4. In an ex vivo settings, such as within an organ bath, the concentration of the spasmogen reaching ASM is readily controlled. This is not quite the case for doses of spasmogen delivered in vivo. The level of ASM activation in vivo depends on the quantity of spasmogen reaching ASM and acting on it simultaneously. This, in turn, depends on the localized spasmogen de position, the permeability of the epithelium and the lamina propria, as well as the rate of clearance from both catabolism and blood circulation [19]. The total level of ASM activation in vivo is also influence by the presence and the concentration of all the endogenous constrictors and dilators acting on the muscle. The force also depends on ASM contractile capacity. So that for any given level of ASM activation, varying levels of force can be developed, 
depending on the size of ASM and its stress-generating capacity (stress is force/cross-sectional area). Second, the level of airway narrowing depends on ASM shortening. During force development, ASM strives to shorten. Owing to its nearly circumferential arrangement around the airways, ASM shortening causes airway narrowing. It addition to the amount of force generated, the level of shortening is controlled by a counterbalancing load. So for a given ASM force, varying level of ASM shortening can occur, depending on the load [20]. The theoretical load impeding ASM shortening in an asthmatic and a non-asthmatic airway of the $10^{\text {th }}$ generation within a lung inflated to a transpulmonary pressure of $5 \mathrm{~cm} \mathrm{H} \mathrm{H}_{2} \mathrm{O}$ is shown in Figure 5 . The intrinsic ability of ASM to shorten, as well as the speed of shortening, can also differ and affect airway narrowing independently of ASM force. Bullimore and coworkers have recently demonstrated that faster shortening can be at least as detrimental as a proportional gain of force to amplify airway narrowing upon ASM stimulation [21]. Third, the level of airway narrowing depends on geometrical factors. So for the same amount of ASM shortening, varying level of narrowing can occur. For example, thicker airway wall amplifies luminal airway narrowing for any given amount of ASM shortening [22]. Collectively, this suggests that narrowing during a $\mathrm{MCh}$ challenge is due to ASM contraction, but excessive narrowing (AHR) can originate from the interaction between muscle and non-muscle factors.

Airway responsiveness is further complicated by the non-linearity between interrelated variables. Airway narrowing is related to ASM shortening at the $2^{\text {nd }}$ power, and airway resistance is inversely related to the radius of the airway lumen at the $4^{\text {th }}$ power. The curvilinear relationships between ASM force and airway narrowing shown in Figure 5 also illustrates the complexity of airway responsiveness.

\section{Transpulmonary Pressure Swings that Stress and Strain}

It is also important to realize that the load impeding ASM shortening is not static. The lung is a dynamic organ that requires substantial and continuous changes of volume to fulfill its ventilatory function. The lung volume changes by approximately $23 \%$ every 5 seconds during tidal breathing at rest. During a deep inspiration (DI) the volume raises by about $170 \%$. These changes of volume also impose oscillatory changes in the luminal volume of the airways. This is mainly because all airways (apart from the trachea and main stem bronchi) are embedded within the lung and are physically attached to the parenchyma. During inspiration, the tethering forces of the parenchyma pull the airways in every direction. Since ASM is arranged nearly circumferentially around the airways, it also gets strained. It was estimated that ASM is strained by $4 \%$ during tidal breathing, $12 \%$ during a sigh and $25 \%$ during a DI [23]. Changes in posture, such as upright vs recumbent (either prone or supine), also change lung volume and geometry, forcing ASM of many airways to operate at different lengths [24]. The changes of ASM length during breathing obviously depend on the stiffness of the airway wall relative to the parenchyma; airway wall stiffness being, to a great extent, function of ASM activation $[25,26]$. Taken together, this suggests that the length of ASM in vivo fluctuates continuously by a varying amount, which depends on the magnitude of the transpulmonary pressure excursions during breathing, the elasticity of the parenchyma, the force of airway-parenchymal interdependence, as well as the muscle and non-muscle components of the airway wall that determine its stiffness.

The stress excursions, and the attendant strain, experienced by ASM in vivo are important to consider because they alter ASM mechanics considerably(reviewed in [27]). Simulation of breathing maneuvers on ex vivo preparations of ASM decrease ASM force when the muscle is held in isometric condition [28] and cause re-lengthening when ASM is subjected to an isotonic or auxotonic load [29,30]. This suggests that the changes of lung volume occurring in vivo due to breathing maneuvers may be protective against excessive airway responsiveness because it decreases ASM contractility. In fact, this is one of the more likely explanations for the well-described bronchodilator effect of DI [31]. Since the bronchodilator effect of DI is blunted in asthma [32], many suggested that a common defect in asthma could lie in a failure to respond appropriately to the dynamic movement of the lungs. The lack of stress transmission to the ASM, a limitation of strain for a given stress, or a lack of decline of force for a given strain, can all alter the bronchodilator control of breathing maneuvers and, thus, enhance airway responsiveness by increasing ASM contractility. It is noteworthy to mention that in a dynamic environment such as the lungs, additional contractile properties of ASM other than force, shortening and velocity of shortening can affect the level of ASM shortening achieved in response to a given stimulus(reviewed in [27]). These contractile properties will not be discussed here but include the intrinsic stiffness of ASM, as well as ASM's abilities to tolerate and recover from oscillating stress.

Whether the stress or the achieved strain is the main determinant for the loss of ASM contractile capacity elicited by breathing maneuvers is still a matter of debate. Both in vivo and ex vivo studies that investigated that question indicate that the strain is better correlated with the loss of force than the stress $[25,33]$. However, high stress with very little strain was also shown to decrease ASM contractility ex vivo [34], and high transpulmonary pressure with very little changes of lung volume was shown to decrease airway responsiveness in vivo [35]. More studies are clearly needed.

As aforementioned, the level of airway wall strain induced by a given stress depends to a great extent on the level of ASM activation. Many report now documented that ASM tone (i.e. tone here refers to a sustained level of ASM activation, whether it is from vagal origin or from the presence of inflammation-derived spasmogens) prevents the bronchodilator effect of simulated breathing maneuvers by attenuating the strain induced by oscillatory stress $[25,34,36,37]$. Airway tone may thus be an important determinant of airway responsiveness. In fact, the level of airway tone measured by the responsiveness to bronchodilator and airway responsiveness measured by a direct challenge usually correlate, both in normal subjects [38] and in subjects afflicted by lung disorders [39]. Airway tone is also enhanced in asthma [40], and can be at the origin of AHR. Airway tone can also affect airway responsiveness by many other means than by attenuating the strain caused by the oscillatory stress of breathing maneuvers. This has been reviewed recently [41].

The bronchodilator effect of breathing maneuvers has important implications with regard to the way airway responsiveness is measured. The decline of FEV1 assessed by spirometry during a direct challenge is likely to be underestimated, at least to some extent, depending on the efficacy of DI to bronchodilate the airways. As aforementioned, FEV1 requires a DI to total lung capacity prior the forced expiratory maneuver. Repeated FEV1 maneuvers during the administration of the dose-response can thus attenuate airway responsiveness considerably. This effect is similar to the different airway response obtained when using different techniques to deliver the MCh. The dosimeter technique requires repetitive DIs and results in an attenuated airway responsiveness compared to the tidal breathing technique, especially in people with the mildest AHR [42]. This has important implications because those are the subjects for whom the confirmation of asthma diagnosis with a positive MCh challenge would be the most useful, 
and they may oftentimes end up with a false-negative result because of the inhibitory effect of DIs [42]. To circumvent this problem, many investigators have opted for the forced oscillation technique (FOT). This technique consists of delivering pressure oscillation at different frequencies into the lung while the subject is breathing normally into a mouth piece connected to the device (reviewed in [43]). The spectral relationship between pressure and airflow at the mouth constitutes the impedance of the respiratory system (Zrs); respiratory system because the impedance stands not only from the impedance of the airways to airflow, but also from the impedance of the lung and the chest wall. Zrs can be dissociated in 2 terms: respiratory system resistance (Rrs) and respiratory system reactance (Xrs). Rrs is the real part of $\mathrm{Z}$; it is the in-phase component of the relationship between pressure and flow. $\mathrm{Xrs}$ is the imaginary part of $\mathrm{Z}$; it is the out-of-phase component of the relationship between pressure and flow. Xrs comprises inertance and elastance, which are two forces acting in opposite direction. While the inertance dominates at high frequencies, elastance dominates at low frequencies. At resonance frequency, i.e. when the scalar values of inertance and elastance are equal, Zrs becomes a good surrogate of Rrs. Since chest wall and lung resistance are not expected to change during a MCh challenge, the changes of Rrs mainly represent the effect of ASM on airway narrowing. The measurement of Zrs at resonance frequency is thus ideal to assess airway responsiveness, i.e. airway narrowing induced by ASM shortening. Thus, one of the advantages of FOT over spirometry is that it discards the confounding effect of repeated DI during the $\mathrm{MCh}$ challenge. An additional advantage of FOT over spirometry is that no maximal voluntary effort is needed. It is thus easier to assess, especially in the youth, elderly and sick patients.

\section{Heterogeneity}

The aforementioned factors affect narrowing within a single airway. However, the signal used to assess airway responsiveness in vivo is measured at the mouth. Therefore, the measured signal embodies the combined effect of individual airways, together with the influence of the lung and chest wall mechanical properties. The first computational models in the early 1990s that have treated the factors influencing airway resistance as stochastic quantities have predicted that simply by increasing variability, airway resistance increases without having to reduce mean airway caliber [44]. This launched the era of airway narrowing heterogeneity. It was since demonstrated that airway narrowing heterogeneity exists in vivo, being greater in asthmatic compared with non-asthmatic subjects [45], and that it contributes to AHR in asthmatics, both in the youth and the elderly $[46,47]$. The search for the identification of factors affecting narrowing heterogeneity has also became a priority. It turns out that the factors affecting heterogeneity are just as numerous as the factors affecting airway narrowing. In addition, the concepts of parallel and axial (in series) interdependence of the different pathways that constitute the tracheobronchial tree were born $[48,49]$. Airflow within the lung is not randomly distributed. More patent airways receive more flow, and vice-versa. Greater airflow down more open airways allows the lung tissue to distend, which then applies traction onto surrounding airways. Conversely, less airflow down more narrowed airways results in non-distention and potentially some degree of collapse of the lung tissue. This reduces distention on surrounding airways. This can have serious implications for the spatial distribution of ventilation. The differential lung traction on the airways is further amplified during bronchoconstriction induced by ASM activation. A small baseline level of heterogeneity between 2 daughter airways directs more flow into the larger one at baseline but ASM activation increases the difference between them. As the level of ASM activation rises, the flow is progressively redistributed toward the more patent airway at the expense of the less patent airway. Consequently, the tethering force of the lung parenchyma that tends to pull the airways open increases in the lung regions subtended by the more patent airways and decreases in the less patent airways. This self-perpetuating feedback loop can eventually reach a critical threshold point where one of the daughter airways dilates despite ASM activation due to the increasing load inflicted by lung recoil, while the other airway collapses due to the combination of ASM activation and decreasing load of the lung recoil. This phenomenon can be at the origin of airway closure [50] and the well-described ventilation defects that characterize asthma [51,52]. The airway generation where flow is seriously compromised due to airway closure or near closure, ultimately determines the size of the ventilation defect and the magnitude the consequent airflow limitation. The number, the size, as well as the spatial localization of ventilation defects affect the degree of airway responsiveness, and likely contribute to asthmatic AHR [53]. So, in addition to the amount of narrowing of individual airways, the pattern of airway constriction within the lung is an important determinant of airway responsiveness. The physiological effects of heterogeneity represent a good example of how a non-muscle factor, which is a small level of heterogeneity between daughter airways, can interact with ASM activation to cause AHR, via increased ventilation distribution heterogeneity. Together, these results suggest that in addition to all the muscle and non-muscle factors that influence individual airway narrowing, the degree of airway narrowing heterogeneity, as well as the spatial arrangement of narrowing within the lung can greatly influence several indices of airway responsiveness.

In addition to provide measurements of lung mechanics during normal tidal breathing that reflect airway caliber, FOT also provide surrogates of ventilation heterogeneity and airway closure [54]. Oscillatory resistance is affected by heterogeneous airway narrowing but elastance is a particularly sensitive measure of ventilation heterogeneity and of airway closure $[55,56]$. Because the FOT provides high temporal resolution of around 5 measurements per second when oscillation frequencies of $5 \mathrm{~Hz}$ are used, the changes within a breath cycle also provide useful information on the dynamic behavior of airways [57]. The FOT measurements are thus sensitive to assess acute changes in the lung, such as the changes occurring during a respiratory maneuver. The changes in airway mechanics at high or low lung volumes may provide useful information on the mechanical properties of the airways that may be related to inflammation, tone and remodeling in asthma $[26,58]$.

Frequency dependence of resistance is also measured by FOT and has been proposed as a way of determining peripheral airway function. This is based on the theory that low oscillation frequencies (between approximately $0.2-8 \mathrm{~Hz}$ ) probe the entire respiratory system from the large airways to the lung periphery. Thus Rrs at $5 \mathrm{~Hz}$ minus Rrs at 20 $\mathrm{Hz}(\Delta \mathrm{R} 5-20)$ is used as a surrogate of peripheral airway resistance, although correlative studies with other measures of peripheral airway function are lacking. There is a suggestion that lung elastance, or lung reactance, which is a composite measure that is dominated by elastance at low oscillation frequencies, is a better measure of heterogeneity than resistance.

Perhaps the most sensitive and specific measure of peripheral airway dysfunction is the multiple breath washout. This is based on the pattern in which alveolar gas leaves the lung. In the presence of small airways disease for example, it takes more breaths for a given amount of alveolar gas to be 'washed out', and there are thus bigger concentration differences of that gas. The tracer gas has to be inert. The 
commonly used gases are nitrogen (washout out by breathing $100 \%$ oxygen), sulphur hexafluoride(SF6) and helium [59]. The washout data are analyzed to determine the severity of ventilation heterogeneity in 2 different peripheral airway compartments. The parameter of ventilation heterogeneity in peripheral airways where gas flow is convection dependent has been named Scond, while heterogeneity at the interface where convective flow changes to diffusion dependent flow (diffusion front) has been named Sacin [60]. Interestingly, Chapman and coworkers have demonstrated that the changes of Sacin are an independent predictor of the increased airway responsiveness to MCh induced by chest wall strapping in healthy (non-asthmatic) subjects [61]. This suggests that, even in the absence of lung pathology, heterogeneous ventilation in the small airways affects airway responsiveness

These complex measurements of peripheral lung and airway physiology in relation to asthma cannot be obtained by conventional spirometry. This is important because it is now recognized that disease of peripheral airways plays an important role in the development of asthma symptoms [62,63]. In fact, there is mounting evidence to suggest that small airway dysfunction, measured by either FOT or multiple-breath nitrogen washout (MBNW) better correlates with asthma symptoms than doesspirometry [64-66]. Certain parameters obtained from FOT and MBNW, such as reactance and Sacin, are the best predictors for the loss of asthma control [67] and are really useful for guiding medication dosage [68]. They are also the best surrogates for monitoring improvement of asthma symptoms that occurred in response to inhaled corticosteroids [64]. These observations reinforce the incentive to scrutinize the factors involved in flow limitation within the small airways. Because of the time resolution of FOT, which is as mentioned above one over the frequency $(1 / f)$, the kinetics of narrowing can also be deduced, which is impossible using spirometry. Combined with lung imaging techniques, FOT and gas washout has the potential to significantly advance our understanding of AHR in asthma [69].

Certainly investigators in this field of research are currently working at an exciting time because of rapidly developing new technologies both in hardware, software and analysis techniques. Our understanding of the pathophysiology of asthmatic AHR will evolve rapidly. The recent progress made has started to decipher the very complex nature of airway responsiveness.

\section{Inflammation and Airway Hyperresponsiveness in Asthma}

A hypothetical scheme showing how inflammation and AHR combine to trigger asthma symptoms is illustrated in Figure 6. On one hand, someone can be afflicted by asthma because of severe inflammation even if hyporesponsive. On the other hand, someone with very little inflammation can be asthmatic because of AHR. The threshold of inflammation that can be tolerated before experiencing asthma symptoms depends on the level of airway responsiveness. So that little airway inflammation, such as the one induced by a common cold for example, is unlikely to cause the appearance of symptoms in normo or hyporesponsive individuals, but likely to cause asthma in hyperresponsive individuals.

Many years of intensive research have unveiled a multifaceted link between asthmatic inflammation and AHR. The purpose of trying to determine whether causality exists between inflammation and AHR is questionable. As highlighted in the previous sections, asthmatic inflammation and AHR are two complex traits that can each originate from a plethora of factors, where every factor can be independent, interconnected and dispensable. It was clearly demonstrated in animal

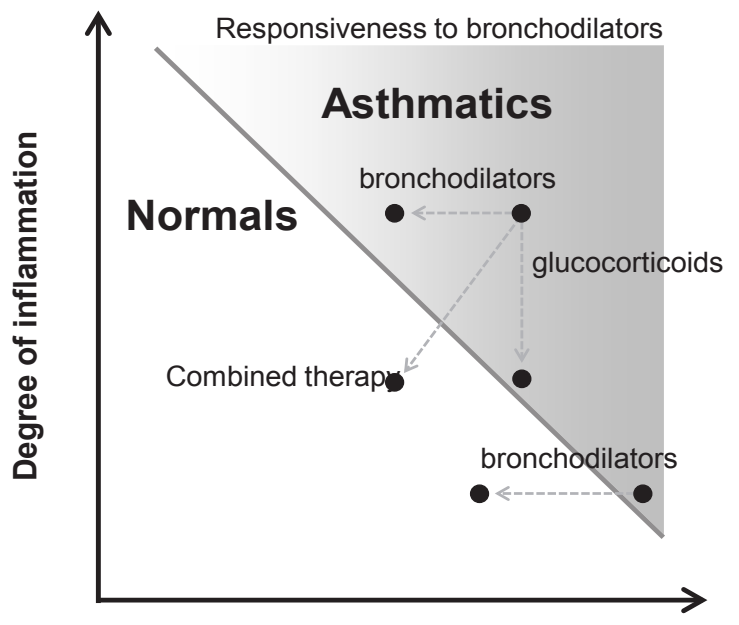

Level of airway responsiveness

Figure 6: Inflammation and airway hyperresponsiveness combine to cause asthma. The diagonal grey line represents the threshold to diagnose asthma. The cutoff obviously varies from one clinician to the other. The cutoff can also vary in time due to the effect of inflammation on airway responsiveness (see variable component of airway responsiveness in the text). During the natural course of asthma, a part of symptoms, which varies in magnitude in between patients, are mediated by the level of airway responsiveness. In turn, the level of airway responsiveness depends on both the amount of inflammationderived spasmogens present, which dictates the level of airway smooth muscle activation and the patient's level of airway responsiveness per se (i.e. responsiveness to any given amount of spasmogens). Whereas someone hyperresponsive will experience symptoms with very little inflammationderived spasmogens, a normo- or hyporesponsive individual will tolerate more inflammation and spasmogens before experiencing symptoms and be diagnosed. The filled circles and the dashed arrows indicate how anyone's asthmatic can be shifted within this frame by the mainstay therapies for asthma. These therapies relieve symptoms by either decreasing the level of airway smooth muscle activation (bronchodilators) or by buffering the inflammation (glucocorticoids). The responsiveness to bronchodilators (grey shaded gradient in asthmatics) depends on the contribution of airway smooth muscle contraction in the elaboration of symptoms. Whereas hyporesponsive patients who suffer asthma predominantly due to severe inflammation are refractory to bronchodilators, patientshyperresponsive with very little inflammation can be fully controlled by bronchodilators alone. It is also important to understand that asthma is not a dichotomic variable. Anyone in the population fits somewhere in this frame. The cutoff for diagnosis is only made to guide clinical practice.

models of asthma that the link between inflammation and airway responsiveness depends on the mouse strain, the environmental trigger used (antigens, LPS, ...), the route of spasmogen delivery (inhaled, intratracheal or intravenous), the measures used to assess both airway responsiveness (airway resistance, elastance, tissue damping, ...) and inflammation (cell counts, cytokines, ...), and the time frame studied between exposure and measurements [70,71]. Therefore, the relationship between inflammation and AHR is conditional. The relationship can also goes in both directions, as airway remodeling induced by allergic airway inflammation elicited by OVA sensitization and challenge in mice was shown to reduce airway responsiveness in some circumstances [72].

In humans, inflammation usually aggravates airway responsiveness to MCh. A review of the literature concluded that glucocorticoids attenuate AHR [73]. There is also evidence that continued usage of low-dose glucocorticoids during the maintenance phase of treatment in mild asthmatics would provide additional improvement on airway responsiveness $[74,75]$. The extent by which glucocorticoids attenuate AHR is such that an important proportion of asthmatics become normoresponsive by the end of the treatments [64]. Glucocorticoids 
are also just as effective to attenuate airway responsiveness in recently diagnosed versus long-standing asthma [76].

Conversely, increasing inflammation artificially by inhaling either interleukin-5 [77] or allergens [78,79] was shown to increase airway responsiveness. Therefore, the degree of airway responsiveness is not fixed (Figure 1). Part of AHR is transiently acquired by inflammation and is variable depending on the severity of inflammation. The other part of AHR is more fixed and can be further divided in 2 parts, an inherent component and an acquired component (Figure 7).

Owing to the many factors influencing airway responsiveness, the choice of spasmogen delivered is also important to consider. Some spasmogens can affect non-muscle factors, which can then interact with ASM shortening to enhance airway responsiveness. Histamine, for example, stimulates mucus hypersecretion and causes edema by increasing vascular permeability [80]. Histamine also has an important influence on many cells of the immune system [81]. Thus, part of the responsiveness to histamine depends on other factors than ASM shortening. The histamine receptor mediating ASM contraction $\left(\mathrm{H}_{1}\right)$ also gets desensitized rapidly [82], which alters by an unpredictable amount the cumulative response. Because of receptor desensitization, the response also depends on the natural state of histamine activation prior the challenge. High doses of histamine can also give important headache. To avoid all these complication, MCh has become the spasmogen of choice to assess airway responsiveness.

\section{Transient Component of AHR}

Inflammation can alter airway responsiveness by increasing vascular permeability, which leads to vascular leakage, edema and thickening of the airway wall. This then increases airway responsiveness by encroaching directly upon the airway lumen, by amplifying luminal narrowing induced by ASM shortening, and by increasing the propensity for closure and ventilation defects. This can certainly occur in the case of cardiac asthma [83]. Mucus hypersecretion and inflammatory cell infiltrates into the airway lumen have the same detrimental consequence as airway wall thickening on airway responsiveness, in addition to increase dangerously the risk of airway plugging. Inflammation can also alter surface tension by many means. Leakage of fibrin in the alveoli, for example, was shown to increase surface tension by degrading the surfactant, resulting in AHR [84]. Inflammation can also increase epithelium permeability by altering the

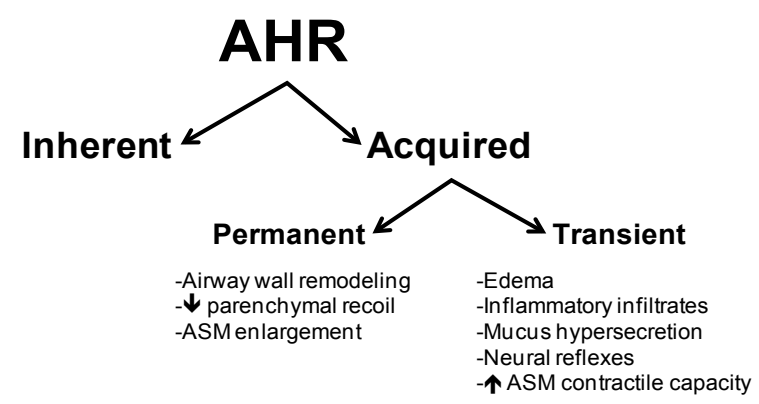

Figure 7: The origin of airway hyperresponsiveness is manifold. The factors that contribute to excessive airway responsiveness can be either inherent or acquired due to inflammatory and remodeling processes that take place in asthmatic lungs. In turns, the acquired defects can be either transient or more permanent. The transient defects are the ones responsive to treatments and are responsible for the variable component of AHR. The permanent defects are more refractory to current asthma therapies, and are responsible for part of the fixed (or hardly modifiable) component of AHR. See text for further detail.

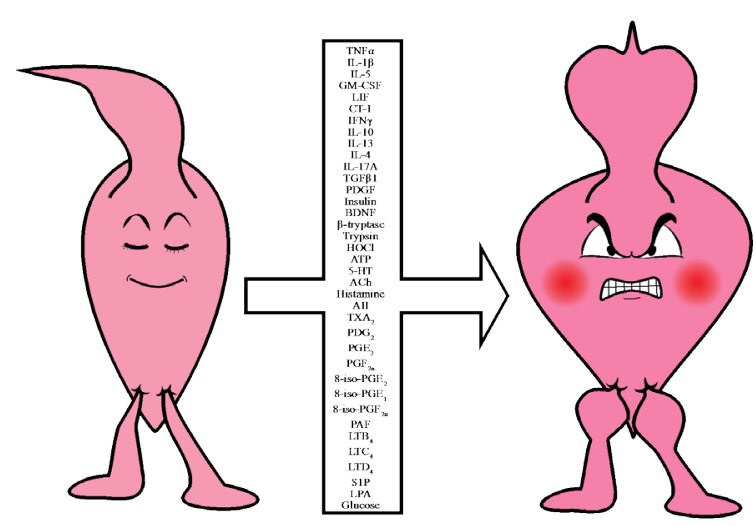

Figure 8: The contractile capacity of ASM is not fixed. Many mediators that are often times dysregulated in asthmatic lungs (enumerated in the middle column) have been shown to increase the contractile capacity of ASM. This suggests that, although heterogeneous between affected individuals, the inflammatory milieu of anyone asthmaticis likely to possess mediators that are capable to render ASM hypercontractile. This acquired hypercontractility can, in turn, contribute to the transient component of AHR [27]

integrity of the adhesion junctions or even by causing desquamation. This then increases airway responsiveness by facilitating the delivery of the inhaled spasmogen to ASM [19]. These are many ways by which inflammation can enhance airway responsiveness, and are all possible reasons explaining the beneficial effect of the glucocorticoids on AHR. Collectively, these results suggest that the transient component of AHR is partially acquired due to the inflammation that characterizes asthma.

The variable component of AHR can also be due to the plasticity of ASM contractile capacity (Figure 8). The capacity of ASM to generate stress (force/cross-sectional area) is not fixed. Dozens of mediators have been shown to increase ASM contractile capacity (reviewed in [27]). The altered expression of those mediators at baseline or during a sudden flare of inflammation can thus increase ASM capacity to generate stress. This type of acquired hypercontractility can certainly contribute to AHR, but is conditional to the presence and the concentration of those mediators. The efficacy of steroids to correct the altered expression of those mediators can also contribute to their ability to reduce AHR.

\section{Permanent Component of AHR that is Inherent}

The non-inflammatory mechanisms of AHR in asthma has been reviewed [18]. The inherent part of AHR depends on the factors discussed above and is hardly modifiable. The level of airway responsiveness is affected by the size and the geometry of the lung and the airways, ASM mass and contractile capacity, lung recoil, heterogeneity of airway narrowing, airway wall thickness and composition, mucus abundance, surface tension, breathing depth and patterns, vascular permeability, force of airway-parenchymal interdependence, transpulmonary pressure, vagal tone, circulating levels of catecholamines and many other factors. Any inherent defects or increased influence of those factors can contribute to AHR. From this point of view, AHR confers risk to suffer from asthma. This is because influx of inflammatory cells and their mediators (including spasmogens) into hyperreactive airways is conducive of asthma symptoms. It was previously thought that glucocorticoids have very limited effect on the permanent component of AHR that is inherent. However, a recent study demonstrates that 14 days of oral prednisolone altered gene expression in ASM, which correlated with improved lung function [85]. This suggests that glucocorticoids may influence airway responsiveness by acting directly on ASM. 
A surprisingly high proportion of the population demonstrates asymptomatic AHR (reviewed in [86]. Approximately half of the subjects with AHR have asthma [87]. Although it could be inherent, it is also possible that asymptomatic AHR is an early sign of asthma development. In fact, a great proportion of individuals with asymptomatic AHR presented with some features of asthma, and a greater proportion of these individuals went on to develop asthma [88]. Clinicians even see it as an opportunity for preventive screening and early interventions [86].

With regard to the inherent ASM capacity to generate stress, no convincing data currently support the notion of greater stressgenerating capacity in asthmatic ASM as a common cause of AHR (reviewed in [89]. So when ASM tissues from asthmatics and nonasthmatics are isolated and compared in ex vivo studies, no consistent difference is observed.

\section{Permanent Component of AHR that is Acquired}

The other fixed part of AHR is acquired and probably the results of long-term effect of inflammation on airway and lung architecture. Inflammation causes damage that can lead to structural remodeling if repair is excessive and/or impaired. These structural changes can amplify narrowing induced by ASM shortening and attenuate the load impeding ASM shortening. They include airway wall thickening, as well as loss of parenchymal recoil and airway-parenchyma interdependence. In fact, remodeling features such as subepithelial fibrosis was shown to correlate with airway responsiveness [90]. From this point of view, AHR is induced by the underlying pathophysiology leading to asthma. Current asthma therapies have a very limited effect on this component of AHR. However, prolonged treatment with glucocorticoids may be beneficial, as the thickness of the reticular basement membrane slowly decreased with long-term intervention with inhaled corticosteroid, and this change correlated temporally with improvement of airway responsiveness [75]. It is worthy to mention that some aspect of remodeling can also attenuate airway responsiveness, such as stiffening of the airway wall [91].

With regard to ASM, the last century of research has consistently shown that ASM mass is enlarged in asthma. A recent study has also demonstrated that narrowing of isolated airways upon ACh stimulation is increased in asthmatics [92]. This seems somewhat counterintuitive to the current notion that the stress-generating capacity of ASM is not different in asthmatics [89], at least not permanently. However, the total force is not only proportional to ASM stress-generating capacity but also to its cross-sectional area. In fact, the same study demonstrated that narrowing of isolated airways correlates with ASM mass [92]. ASM enlargement may thus contribute to AHR. The phenomena and individual mediators that may contribute to ASM enlargement in asthma have been reviewed many times $[93,94]$.

\section{Improving Asthma Care one more Notch}

As we come to realize that the number of factors potentially contributing to AHR increases, it implies that their respective contribution within a group of asthmatics inevitably wanes. This certainly tarnishes the faith of people that think that targeting a single factor can prove beneficial in the treatment of a group of patients suffering from a lung disorder as heterogeneous as asthma.

To improve the quality of life of patients suffering from asthma beyond the benefits provided by broad-spectrum anti-inflammatory drugs, such as glucocorticoids, and broad spectrum ASM relaxant, such as the bronchodilators, research and clinical scientists need to lean towards personalized medicine. The lack of breakthroughs in the development of asthma treatments that has plagued the last few decades of research can partially be attributed to the way we are trained to do science; i.e., comparing groups of treated and non-treated individuals to demonstrate statistically significant results. Now that we know that asthma is characterized by many phenotypes and endotypes, this approach may no longer provide valuable information. Owing to the very distinct factors that can lead to asthma, targeting a single factor within an entire cohort of asthmatics is more likely to be insignificant. Individualized therapy is a more promising approach. Targeting a single factor in an affected individual can be salutary if this specific factor contributes to inflammation, AHR or both within this individual. It does not exclude the complexity and the numerous factors that can be involved within a single individual, but at least eliminates the variability due to the distinctive factors involved in between individual. Plus, contributing factors oftentimes act synergistically with other defects to aggravate asthma. Thus, blocking a contributing factor within an individual may not only subtract its independent effect, but also the synergistic effects it maintains with the other defects. Therefore, the hope is still present that targeting a single contributing factor within an individual, but not within a group, may be worthy.

A growing body of literature now documents the many ways by which airway responsiveness can be dysregulated by inflammation in asthma. The time has arrived for creative scientists to use this growing bulk of knowledge and translate it to clinical care. We think that we should henceforth emphasize on extending the characterization of the patients once the diagnosis of asthma is confirmed. Thereby, the contributing factors causing both inflammation and AHR within the affected individuals can be identified and then targeted.

\section{Conclusions}

The origin of increased airway responsiveness is difficult to infer. The last decades of research have started to unfold the multifaceted link between inflammation and AHR in asthma. On one hand, AHR can be an important predisposing factor for asthma once an individual is beset with airway inflammation. On the other hand, many aspects of asthmatic inflammation have the potential to alter the level of airway responsiveness and to be responsible for either the appearance or the worsening of AHR. Part of the acquired component of AHR is due to ASM plasticity. The mass and the stress-generating capacity of ASM are not fixed. The recognition that a growing body of muscle and nonmuscle factors, as well as their interaction, can contribute to AHR in only some of the affected individuals is a stepping stone heading in the right direction. The hope is that this better understanding will eventually avail patients.

\section{References}

1. Bossé Y, Hudson TJ (2007) Toward a comprehensive set of asthma susceptibility genes. Annu Rev Med 58: 171-184.

2. Boyce JA, Bochner B, Finkelman FD, Rothenberg ME (2012) Advances in mechanisms of asthma, allergy, and immunology in 2011. J Allergy Clin Immunol 129: 335-341.

3. Papadopoulos NG, Christodoulou I, Rohde G, Agache I, Almqvist C, et al. (2011) Viruses and bacteria in acute asthma exacerbations--a GA ${ }^{2}$ LEN-DARE systematic review. Allergy 66: 458-468.

4. Laumbach RJ, Kipen HM (2012) Respiratory health effects of air pollution: update on biomass smoke and traffic pollution. J Allergy Clin Immunol 129: 3-11.

5. Khan DA (2012) Exercise-induced bronchoconstriction: burden and prevalence Allergy Asthma Proc 33: 1-6. 
Citation: Bossé Y, Lee-Gosselin A, Boulet LP, King GG (2013) Airway Hyperresponsiveness in Asthma: A Better Understanding Yet to Yield Clinical Benefit. J Allergy Ther 4: 150. doi:10.4172/2155-6121.1000150

6. Brooks SM, Bernstein IL (2011) Irritant-induced airway disorders. Immunol Allergy Clin North Am 31: 747-768.

7. Labrecque M (2012) Irritant-induced asthma. Curr Opin Allergy Clin Immunol 12: $140-144$

8. Quirce S, Bernstein JA (2011) Old and new causes of occupational asthma. Immunol Allergy Clin North Am 31: 677-698.

9. Anderson SD (2010) Indirect challenge tests: Airway hyperresponsiveness in asthma: its measurement and clinical significance. Chest 138: 25S-30S.

10. Cockcroft DW (2010) Direct challenge tests: Airway hyperresponsiveness in asthma: its measurement and clinical significance. Chest 138: 18S-24S.

11. Holgate ST (2011) Asthma: a simple concept but in reality a complex disease. Eur J Clin Invest 41: 1339-1352.

12. Manso L, Madero MF, Ruiz-García M, Fernández-Nieto M, Sastre J (2011) Comparison of bronchial hyperresponsiveness to methacholine and adenosine and airway inflammation markers in patients with suspected asthma. J Asthma 48: $335-340$

13. Boulet LP, Gauvreau G, Boulay ME, O'Byrne P, Cockcroft DW; Clinical Investigative Collaboration, et al. (2007) The allergen bronchoprovocation model: an important tool for the investigation of new asthma anti-inflammatory therapies. Allergy 62: 1101-1110.

14. Brutsche MH, Downs SH, Schindler C, Gerbase MW, Schwartz J, et al. (2006) Bronchial hyperresponsiveness and the development of asthma and COPD in asymptomatic individuals: SAPALDIA cohort study. Thorax 61: 671-677.

15. Leuppi JD, Salome CM, Jenkins CR, Anderson SD, Xuan W, et al. (2001) Predictive markers of asthma exacerbation during stepwise dose reduction of inhaled corticosteroids. Am J Respir Crit Care Med 163: 406-412.

16. O'Connor G, Sparrow D, Taylor D, Segal M, Weiss S (1987) Analysis of doseresponse curves to methacholine. An approach suitable for population studies. Am Rev Respir Dis 136: 1412-1417.

17. Salome CM, Brown NJ, Reddel HK, Xuan W, Marks GB (2011) Indices of bronchial reactivity and sensitivity. Thorax 66: 265-266.

18. Baroffio M, Barisione G, Crimi E, Brusasco V (2009) Noninflammatory mechanisms of airway hyper-responsiveness in bronchial asthma: an overview. Ther Adv Respir Dis 3: 163-174.

19. Bates JH, Stevenson CA, Aliyeva M, Lundblad LK (2012)Airway responsiveness depends on the diffusion rate of methacholine across the airway wall. J Appl Physiol (1985) 112: 1670-1677.

20. Paré PD (2003) Airway hyperresponsiveness in asthma: geometry is not everything! Am J Respir Crit Care Med 168: 913-914.

21. Bullimore SR, Siddiqui S, Donovan GM, Martin JG, Sneyd J, et al. (2011) Could an increase in airway smooth muscle shortening velocity cause airway hyperresponsiveness? Am J Physiol Lung Cell Mol Physiol 300: L121-131.

22. Paré PD, McParland BE, Seow CY (2007) Structural basis for exaggerated airway narrowing. Can J Physiol Pharmacol 85: 653-658.

23. Fredberg JJ, Inouye D, Miller B, Nathan M, Jafari S, et al. (1997) Airway smooth muscle, tidal stretches, and dynamically determined contractile states. Am J Respir Crit Care Med 156: 1752-1759.

24. Tawhai MH, Nash MP, Lin CL, Hoffman EA (2009) Supine and prone differences in regional lung density and pleural pressure gradients in the human lung with constant shape. J Appl Physiol (1985) 107: 912-920.

25. Noble PB, McFawn PK, Mitchell HW (2007) Responsiveness of the isolated airway during simulated deep inspirations: effect of airway smooth muscle stiffness and strain. J Appl Physiol (1985) 103: 787-795.

26. Kelly VJ, Brown NJ, Sands SA, Borg BM, King GG, et al. (2012) Effect of airway smooth muscle tone on airway distensibility measured by the forced oscillation technique in adults with asthma. J Appl Physiol (1985) 112: 1494-1503.

27. Bossé Y, Paré PD (2013) The contractile properties of airway smooth muscle: How their defects can be linked to asthmatic airway hyperresponsiveness? Curr Respir Med Rev 9: 42-68.

28. Gunst SJ (1983) Contractile force of canine airway smooth muscle during cyclical length changes. J Appl Physiol Respir Environ Exerc Physiol 55: 759769.

29. Oliver MN, Fabry B, Marinkovic A, Mijailovich SM, Butler JP, et al. (2007) Airway hyperresponsiveness, remodeling, and smooth muscle mass: right answer wrong reason? Am J Respir Cell Mol Biol 37: 264-272.

30. Mitchell RW, Dowell ML, Solway J, Lakser OJ (2008) Force fluctuation-induced relengthening of acetylcholine-contracted airway smooth muscle. Proc Am Thorac Soc 5: 68-72.

31. NADEL JA, TIERNEY DF (1961) Effect of a previous deep inspiration on airway resistance in man. J Appl Physiol 16: 717-719.

32. Salome CM, Thorpe CW, Diba C, Brown NJ, Berend N, et al. (2003) Airway renarrowing following deep inspiration in asthmatic and nonasthmatic subjects. Eur Respir J 22: 62-68.

33. Salerno FG, Pellegrino R, Trocchio G, Spanevello A, Brusasco V, et al. (2005) Attenuation of induced bronchoconstriction in healthy subjects: effects of breathing depth. J Appl Physiol (1985) 98: 817-821.

34. Pascoe CD, Seow CY, Paré PD, Bossé Y (2013) Decrease of airway smooth muscle contractility induced by simulated breathing maneuvers is not simply proportional to strain. J Appl Physiol (1985) 114: 335-343

35. Duggan CJ, Chan J, Whelan AJ, Berend N (1990) Bronchodilatation induced by deep breaths in relation to transpulmonary pressure and lung volume. Thorax 45: 930-934.

36. Lavoie TL, Krishnan R, Siegel HR, Maston ED, Fredberg JJ, et al. (2012) Dilatation of the constricted human airway by tidal expansion of lung parenchyma. Am J Respir Crit Care Med 186: 225-232.

37. Noble PB, Jones RL, Needi ET, Cairncross A, Mitchell HW, et al. (2011) Responsiveness of the human airway in vitro during deep inspiration and tidal oscillation. J Appl Physiol (1985) 110: 1510-1518.

38. Bergner A, Kellner J, Kemp da Silva A, Fischer R, Gamarra F, et al. (2006) Bronchial hyperreactivity is correlated with increased baseline airway tone. Eu J Med Res 11: 77-84

39. Benson MK (1978) Bronchial responsiveness to inhaled histamine and isoprenaline in patients with airway obstruction. Thorax 33: 211-213.

40. Molfino NA, Slutsky AS, Julià-Serdà G, Hoffstein V, Szalai JP, et al. (1993) Assessment of airway tone in asthma. Comparison between double lung transplant patients and healthy subjects. Am Rev Respir Dis 148: 1238-1243.

41. Bosse Y, Pare PD, Bosse Y (2012) Airway Smooth Muscle in Asthma Symptoms: Culprit but Maybe Innocent In: Irusen EM (Ed.), Lung Diseases - Selected State of the Art Reviews: InTech: 3-54.

42. Allen ND, Davis BE, Hurst TS, Cockcroft DW (2005) Difference between dosimeter and tidal breathing methacholine challenge: contributions of dose and deep inspiration bronchoprotection. Chest 128: 4018-4023.

43. Kaczka DW, Dellacá RL (2011) Oscillation mechanics of the respiratory system applications to lung disease. Crit Rev Biomed Eng 39: 337-359.

44. Bates JH (1993) Stochastic model of the pulmonary airway tree and its implications for bronchial responsiveness. J Appl Physiol (1985) 75: 2493-2499.

45. King GG, Carroll JD, Müller NL, Whittall KP, Gao M, et al. (2004) Heterogeneity of narrowing in normal and asthmatic airways measured by HRCT. Eur Respir J 24: 211-218.

46. Hardaker KM, Downie SR, Kermode JA, Farah CS, Brown NJ, et al. (2011) Predictors of airway hyperresponsiveness differ between old and young patients with asthma. Chest 139: 1395-1401

47. Downie SR, Salome CM, Verbanck S, Thompson B, Berend N, et al. (2007) Ventilation heterogeneity is a major determinant of airway hyperresponsiveness in asthma, independent of airway inflammation. Thorax 62: 684-689.

48. Venegas JG, Winkler T, Musch G, Vidal Melo MF, Layfield D, et al. (2005) Selforganized patchiness in asthma as a prelude to catastrophic shifts. Nature 434 777-782.

49. Winkler T, Venegas JG (2007) Complex airway behavior and paradoxical responses to bronchoprovocation. J Appl Physiol (1985) 103: 655-663.

50. King GG, Eberl S, Salome CM, Meikle SR, Woolcock AJ (1997) Airway closure measured by a technegas bolus and SPECT. Am J Respir Crit Care Med 155 682-688.

51. King GG, Eberl S, Salome CM, Young IH, Woolcock AJ (1998) Differences in airway closure between normal and asthmatic subjects measured with singlephoton emission computed tomography and technegas. Am J Respir Crit Care Med 158: 1900-1906. 
Citation: Bossé Y, Lee-Gosselin A, Boulet LP, King GG (2013) Airway Hyperresponsiveness in Asthma: A Better Understanding Yet to Yield Clinical Benefit. J Allergy Ther 4: 150. doi:10.4172/2155-6121.1000150

Page 10 of 10

52. Tzeng YS, Lutchen K, Albert M (2009) The difference in ventilation heterogeneity between asthmatic and healthy subjects quantified using hyperpolarized $3 \mathrm{He}$ MRI. J Appl Physiol (1985) 106: 813-822.

53. Farrow CE, Salome CM, Harris BE, Bailey DL, Bailey E, et al. (2012) Airway closure on imaging relates to airway hyperresponsiveness and peripheral airway disease in asthma. J Appl Physiol (1985) 113: 958-966.

54. Downie SR, Salome CM, Verbanck S, Thompson BR, Berend N, et al. (2013) Effect of methacholine on peripheral lung mechanics and ventilation heterogeneity in asthma. J Appl Physiol (1985) 114: 770-777.

55. Lutchen KR, Gillis H (1997) Relationship between heterogeneous changes in airway morphometry and lung resistance and elastance. J Appl Physiol (1985) 83: $1192-1201$

56. Thorpe CW, Bates JH (1997) Effect of stochastic heterogeneity on lung impedance during acute bronchoconstriction: a model analysis. J Appl Physio (1985) 82: 1616-1625.

57. Dellacà RL, Duffy N, Pompilio PP, Aliverti A, Koulouris NG, et al. (2007) Expiratory flow limitation detected by forced oscillation and negative expiratory pressure. Eur Respir J 29: 363-374.

58. Brown NJ, Salome CM, Berend N, Thorpe CW, King GG (2007) Airway distensibility in adults with asthma and healthy adults, measured by forced oscillation technique. Am J Respir Crit Care Med 176: 129-137.

59. Robinson PD, Latzin P, Verbanck S, Hall GL, Horsley A, et al. (2013) Consensus statement for inert gas washout measurement using multiple- and singlebreath tests. Eur Respir J 41: 507-522.

60. Verbanck S, Paiva M (1990) Model simulations of gas mixing and ventilation distribution in the human lung. J Appl Physiol (1985) 69: 2269-2279.

61. Chapman DG, Berend N, Horlyck KR, King GG, Salome CM (1985) Does increased baseline ventilation heterogeneity following chest wall strapping predispose to airway hyperresponsiveness? J Appl Physiol 113: 25-30.

62. van den Berge M, ten Hacken NH, van der Wiel E, Postma DS (2013) Treatment of the bronchial tree from beginning to end: targeting small airway inflammation in asthma. Allergy 68: 16-26.

63. Thompson BR, Douglass JA, Ellis MJ, Kelly VJ, O'Hehir RE, et al. (2013) Peripheral lung function in patients with stable and unstable asthma. J Allergy Clin Immunol 131: 1322-1328.

64. Farah CS, King GG, Brown NJ, Downie SR, Kermode JA, et al. (2012) The role of the small airways in the clinical expression of asthma in adults. J Allergy Clin Immunol 129: 381-387, 387.

65. Takeda T, Oga T, Niimi A, Matsumoto H, Ito I, et al. (2010) Relationship between small airway function and health status, dyspnea and disease control in asthma. Respiration 80: 120-126.

66. Shi Y, Aledia AS, Tatavoosian AV, Vijayalakshmi S, Galant SP, et al. (2012) Relating small airways to asthma control by using impulse oscillometry in children. J Allergy Clin Immunol 129: 671-678.

67. Shi Y, Aledia AS, Galant SP, George SC (2013) Peripheral airway impairment measured by oscillometry predicts loss of asthma control in children. J Allergy Clin Immunol 131: 718-723.

68. Farah CS, King GG, Brown NJ, Peters MJ, Berend N, et al. (2012) Ventilation heterogeneity predicts asthma control in adults following inhaled corticosteroid dose titration. J Allergy Clin Immunol 130: 61-68.

69. LaPrad AS, Lutchen KR (2008) Respiratory impedance measurements for assessment of lung mechanics: focus on asthma. Respir Physiol Neurobiol 163: 64-73.

70. Janssen-Heininger YM, Irvin CG, Scheller EV, Brown AL, Kolls JK, et al. (2012) Airway Hyperresponsiveness and Inflammation: Causation, Correlation, or No Relation? J Allergy Ther 2012

71. Riesenfeld E, Allen GB, Bates JH, Poynter ME, Wu M, et al. (2012) The Temporal Evolution of Airways Hyperresponsiveness and Inflammation. J Allergy Ther 1: 1-7.

72. Alcorn JF, Rinaldi LM, Jaffe EF, van Loon M, Bates JH, et al. (2007) Transforming growth factor-beta1 suppresses airway hyperresponsiveness in allergic airway disease. Am J Respir Crit Care Med 176: 974-982.

73. Brannan JD (2010) Bronchial hyperresponsiveness in the assessment of asthma control: Airway hyperresponsiveness in asthma: its measurement and clinical significance. Chest 138: 11S-17S.
74. Boulet LP, Turcotte $H$, Prince $P$, Lemière $C$, Olivenstein R, et al. (2009) Benefits of low-dose inhaled fluticasone on airway response and inflammation in mild asthma. Respir Med 103: 1554-1563.

75. Ward C, Pais M, Bish R, Reid D, Feltis B, et al. (2002) Airway inflammation, basement membrane thickening and bronchial hyperresponsiveness in asthma. Thorax 57: 309-316

76. Boulet LP, Turcotte H, Laviolette M, Naud F, Bernier MC, et al. (2000) Airway hyperresponsiveness, inflammation, and subepithelial collagen deposition in recently diagnosed versus long-standing mild asthma. Influence of inhaled corticosteroids. Am J Respir Crit Care Med 162: 1308-1313.

77. Shi HZ, Xiao CQ, Zhong D, Qin SM, Liu Y, et al. (1998) Effect of inhaled interleukin-5 on airway hyperreactivity and eosinophilia in asthmatics. Am J Respir Crit Care Med 157: 204-209.

78. Cockcroft DW, Ruffin RE, Dolovich J, Hargreave FE (1977) Allergen-induced increase in non-allergic bronchial reactivity. Clin Allergy 7: 503-513.

79. Mapp CE, Polato R, Maestrelli P, Hendrick DJ, Fabbri LM (1985) Time course of the increase in airway responsiveness associated with late asthmatic reactions to toluene diisocyanate in sensitized subjects. J Allergy Clin Immunol 75: 568-572.

80. Walsh GM (2002) Second-generation antihistamines in asthma therapy: is there a protective effect? Am J Respir Med 1: 27-34.

81. O'Mahony L, Akdis M, Akdis CA (2011) Regulation of the immune response and inflammation by histamine and histamine receptors. J Allergy Clin Immunol 128: $1153-1162$

82. Krzanowski JJ, Anderson WH, Polson JB, Szentivanyi A (1980) Prostaglandin mediated histamine tachyphylaxis in subhuman primate tracheal smooth muscle. Arch Int Pharmacodyn Ther 247: 155-162.

83. Cabanes LR, Weber SN, Matran R, Regnard J, Richard MO, et al. (1989) Bronchial hyperresponsiveness to methacholine in patients with impaired left ventricular function. N Engl J Med 320: 1317-1322.

84. Wagers SS, Norton RJ, Rinaldi LM, Bates JH, Sobel BE, et al. (2004) Extravascular fibrin, plasminogen activator, plasminogen activator inhibitors, and airway hyperresponsiveness. J Clin Invest 114: 104-111.

85. Yick CY, Zwinderman AH, Kunst PW, Günberg K, Mauad T, et al. (2013) Glucocorticoid-induced changes in gene expression of airway smooth muscle in patients with asthma. Am J Respir Crit Care Med 187: 1076-1084.

86. Boulet LP (2003) Asymptomatic airway hyperresponsiveness: a curiosity or an opportunity to prevent asthma? Am J Respir Crit Care Med 167: 371-378.

87. Woolcock AJ, Peat JK, Salome CM, Yan K, Anderson SD, et al. (1987) Prevalence of bronchial hyperresponsiveness and asthma in a rural adult population. Thorax 42: 361-368.

88. Laprise C, Laviolette M, Boutet M, Boulet LP (1999) Asymptomatic airway hyperresponsiveness: relationships with airway inflammation and remodelling Eur Respir J 14: 63-73.

89. Wright D, Sharma P, Ryu MH, Rissé PA, Ngo M, et al. (2013) Models to study airway smooth muscle contraction in vivo, ex vivo and in vitro: implications in understanding asthma. Pulm Pharmacol Ther 26: 24-36.

90. Boulet LP, Laviolette M, Turcotte H, Cartier A, Dugas M, et al. (1997) Bronchia subepithelial fibrosis correlates with airway responsiveness to methacholine. Chest 112: 45-52.

91. Milanese M, Crimi E, Scordamaglia A, Riccio A, Pellegrino R, et al. (2001) On the functional consequences of bronchial basement membrane thickening. J Appl Physiol (1985) 91: 1035-1040.

92. Noble PB, Jones RL, Cairncross A, Elliot JG, Mitchell HW, et al. (2013) Airway narrowing and bronchodilation to deep inspiration in bronchial segments from subjects with and without reported asthma. J Appl Physiol (1985) 114: 14601471

93. Black JL, Panettieri RA Jr, Banerjee A, Berger P (2012) Airway smooth muscle in asthma: just a target for bronchodilation? Clin Chest Med 33: 543-558.

94. Ozier A, Allard B, Bara I, Girodet PO, Trian T, et al. (2011) The pivotal role of airway smooth muscle in asthma pathophysiology. J Allergy (Cairo)

95. Lambert RK, Wiggs BR, Kuwano K, Hogg JC, Paré PD (1993) Functiona significance of increased airway smooth muscle in asthma and COPD. J App Physiol (1985) 74: 2771-2781. 